\title{
Un juego como estrategia educativa para el control de Aedes aegypti en escolares venezolanos
}

\author{
Edison Vivas ${ }^{1}$ y Milady Guevara de Sequeda ${ }^{2}$
}

RESUMEN Objetivo. Evaluar el juego conocido por Jugando en salud: dengue, como medio de enseñanza-aprendizaje que favorece la incorporación de los escolares de la segunda etapa de educación básica a las actividades de control del mosquito Aedes aegypti y de prevención del dengue en el municipio de Girardot, Estado Aragua, Venezuela.

Métodos. Se efectuó un estudio descriptivo cuasiexperimental basado en una investigación de campo realizada en 9 escuelas seleccionadas por muestreo aleatorio sistemático entre las 29 escuelas estatales del municipio de Girardot, Estado Aragua, Venezuela, que tenían tres o más salones de sexto grado de primaria. En cada escuela se constituyeron tres salones de trabajo: en el grupo de salones no. 1 (210 estudiantes) se practicó el juego tres veces por semana durante 60 días y al maestro se le entregó un material teórico sobre el dengue; en el grupo de salones no. 2 (196 estudiantes) se utilizó solo el material teórico que se le entregó al maestro, y en el grupo no. 3 o grupo de control (215 estudiantes) se siguió la programación habitual establecida por el Ministerio de Educación. A los escolares participantes se les realizó una evaluación antes y después de aplicar el programa propuesto, con un cuestionario que fue evaluado mediante una escala de Likert. Se calcularon las medidas de concentración y dispersión de los datos obtenidos durante las evaluaciones. Se empleó el análisis de la varianza de clasificación simple para comparar los promedios de los datos obtenidos en los distintos salones y detectar diferencias significativas entre los grupos en cuanto a conocimientos y destrezas antes y después de aplicar el programa propuesto. Se utilizó la prueba de Scheffé para determinar diferencias dentro de los grupos.

Resultados. En la investigación participaron 621 escolares de 8 a 16 años de edad (media: 11,8 años). De los 210 estudiantes que participaron en el juego, 41\% lo valoraron "de alta aceptación" y 45\% "de muy alta aceptación". Los conocimientos sobre el dengue y las destrezas (conjunto de habilidades adquiridas) medidos antes de aplicar el programa previsto fueron inferiores (6,5 y 18,4 puntos, respectivamente) a los obtenidos en la prueba final (8,25 y 22,9 puntos, respectivamente; $\mathrm{P}<0,05)$. Se evidenció un mayor aprendizaje en los grupos que utilizaron el juego más el material teórico (grupos de salones no. 1) y el material teórico solamente (grupo de salones no. 2) que en el grupo de control (grupo de salones no. 3) $(\mathrm{P}<$ 0,05). También se encontró que los estudiantes de los grupos de salones nos. 1 y 2 incorporaron más destrezas y habilidades que los del grupo de control $(\mathrm{P}<0,05)$.

Conclusiones. El juego tuvo muy buena aceptación entre los estudiantes de la segunda etapa de la enseñanza básica. Les permitió adquirir más conocimientos sobre el dengue y desarrollar habilidades y destrezas conducentes a su incorporación en actividades para la prevención de la enfermedad en sus respectivas comunidades. Se recomienda extender este juego a todas las escuelas básicas como una vía para fortalecer el proceso educativo e incorporar a los niños a las actividades de control del dengue.

Palabras clave Juegos experimentales, materiales de enseñanza, dengue.

1 Ministerio de Salud y Desarrollo Social, Dirección de Contraloría Sanitaria y Saneamiento Ambiental, Maracay, Venezuela. Dirección postal: Urbanización
San Ignacio, Calle Tachira cruce con Calle Zulia No. 2-B, Municipio Girardot, Parroquia José Casanova Godoy, Maracay, Estado Aragua, Venezuela.
2 Universidad de Carabobo, Facultad de Ciencias de la Salud, Núcleo Aragua, Maracay, Venezuela. 
La actividad lúdica contribuye al desarrollo físico y social del niño, facilita su desarrollo motor e intelectual y mejora su capacidad de concentración, percepción y memoria, por lo que constituye un pilar básico para su educación. ${ }^{3}$ Como medio educativo, el juego ayuda al individuo a adaptarse a diversas situaciones en la vida real.

Desde el punto de vista biológico y psicológico, los juegos intervienen en la formación del carácter del preadolescente, enseñándole a tomar decisiones, luchar por un objetivo, aceptar la derrota o lograr el éxito con respeto y espíritu de decisión. ${ }^{4}$ En los escolares, el juego ayuda a comprender, penetrar y captar el mundo circundante, a formar sentimientos y a reafirmar prerrogativas y respeto, utilizando para ello un lenguaje propio.

El valor didáctico del juego ha sido estudiado por muchos autores, pero Froebel fue el primero en reconocer su valor educativo y en incorporarlo a la pedagogía. Según este autor, el juego es, a un mismo tiempo, modelo y reproducción de la vida y debe ser parte esencial de toda educación, por constituir para el niño una función natural (1).

La educación debe ser un proceso sistemático dirigido a fomentar cambios en la conducta de los alumnos mediante un aprendizaje duradero y positivo (2). Para conseguir esos cambios se debe prestar atención a las diferentes etapas de este proceso: la definición de los objetivos, el ordenamiento de las estrategias metodológicas, el aprendizaje, la evaluación y el reajuste. De ellas, el ordenamiento de las estrategias metodológicas para el aprendizaje y la evaluación constituyen la base operativa de la educación.

La eficacia de las estrategias metodológicas depende de la ca-

\footnotetext{
3 Bolívar G. El juego en la primera etapa de la escuela básica. Universidad Pedagógica Experimental Libertador. Maracay, Estado Aragua, Venezuela. Trabajo de ascenso no publicado; 1993.

4 Rivas C. El tiempo libre y el ocio en la educación preescolar y básica. Universidad del Zulia, Maracaibo, Estado Zulia, Venezuela. Trabajo de ascenso no publicado; 1986.
}

pacidad del educador para aplicarlas de acuerdo con el objetivo buscado. Cuando el proceso educativo está orientado a cultivar actitudes y prácticas saludables, las estrategias más apropiadas son las que permiten la interacción y el diálogo, así como la observación y la experimentación. Mediante ellas los educandos desarrollan su propia capacidad para argumentar y determinar su comportamiento (3).

La evaluación, por otra parte, se centra en actividades y procesos orientados a la acción. De ahí que, según el enfoque constructivista, ${ }^{5}$ un programa educativo o de aprendizaje deba dirigirse a solucionar un problema social o individual importante en un contexto natural y una situación real concreta. Las acciones desarrolladas deben ser factibles, eficaces y eficientes si han de lograr los resultados propuestos (4).

En Venezuela, la educación básica está orientada a ofrecer una formación integral que garantice a los ciudadanos el desarrollo de aptitudes para desenvolverse en la sociedad actual. En este contexto, el juego es importante como recurso didáctico innovador, sobre todo en el contexto de las ciencias naturales, ya que permite integrar elementos del ambiente con conceptos básicos como el de enfermedad y prevención - de una manera didáctica, original y participativa (5, 6).

En las escuelas se han puesto en marcha diversos programas para lograr el cambio de conducta de niños y jóvenes en relación con la salud. Los educadores en materia de salud tienen ante sí el gran reto de lograr que el proceso de enseñanza y aprendizaje en las escuelas influya palpablemente en la conducta sanitaria de los estudiantes, haciéndole contrapeso al entorno informativo y valorativo del niño fuera de la escuela, que puede ser tan

\footnotetext{
El enfoque constructivista en la enseñanza se apoya en el concepto de la enseñanza activa, que tiende a favorecer el razonamiento científico y que contrasta con la visión pasiva tradicional de otros modelos de aprendizaje. El constructivismo postula que toda persona construye su propio conocimiento tomando de su ambiente los elementos que su propia estructura cognoscitiva sea capaz de asimilar (6).
}

importante como el mensaje escolar o incluso más $(6,7)$.

El Ministerio de Educación de Venezuela ha definido la evaluación del aprendizaje como un proceso de investigación que recoge y analiza pruebas de lo que acontece en el acto de enseñanza-aprendizaje. Aunque mantiene el énfasis en lo cualitativo, utiliza métodos cuantitativos para lograr una visión más completa y profunda que la obtenida por ambos métodos por separado.

En América Latina, los juegos se han utilizado como parte de estrategias de enseñanza para promover el control y la prevención de enfermedades. No obstante, son pocas las investigaciones publicadas en este sentido. Santos y colaboradores utilizaron en 1993 juegos para enseñarles a jóvenes y adolescentes a disminuir la prevalencia de enfermedades, tales como la esquistosomiasis, el sida, el dengue y la leishmaniasis (8). Dos años después, Soto y colaboradores publicaron los resultados de la evaluación de un programa educativo sobre el dengue y Aedes aegypti en el cual se observaron cambios en la actitud de las personas hacia la enfermedad (9). Estas experiencias confirman la importancia del juego como elemento educativo y la utilidad de su aplicación práctica en la educación sanitaria.

Desde el punto de vista teórico, la importancia del juego no solo está dada por el tiempo que los niños le dedican, sino por las implicaciones que tiene en todas las áreas del comportamiento humano (10). El juego y el impulso de jugar están asociados con los actos y problemas de la vida; el juego es una necesidad vital cuyo origen debe buscarse en una serie de impulsos que se van generando gradualmente hasta adquirir una forma determinada bajo la influencia del medio circundante (11).

Según Díaz Vega (10), Bandura, Piaget y Freud coinciden en pensar que, para los niños, los juegos constituyen modelos para la adquisición de nuevos patrones de respuestas, sobre todo cuando semejan situaciones de la vida real. Estos autores también consideran que el juego es fundamental en el desarrollo del niño debido a las vivencias que este adquiere y a la posibilidad que le ofrece de regular las 
tensiones creadas por los adultos e imitar conductas, todo lo cual estimula su capacidad cognoscitiva. El juego a base de reglas desempeña un papel especialmente importante en la socialización del niño y puede utilizarse para afianzar conceptos (carácter competitivo), adquirir destrezas (carácter repetitivo), enseñar a tomar decisiones y a cumplir instrucciones, establecer hábitos de comportamiento e higiene, y reforzar el proceso constructor del pensamiento.

La legislación venezolana ha promovido la aplicación de los juegos como parte de la estrategia educativa. Esto se refleja en la Constitución de la República Bolivariana de Venezuela (artículo 102) y en la Ley Orgánica para la Protección del Niño y el Adolescente (artículo 63), donde se establece que la finalidad de la educación es desarrollar el potencial creativo de cada ser humano y su participación activa, consciente y solidaria, y que el Estado "debe satisfacer las diferentes necesidades e intereses de los niños y adolescentes y fomentar los juguetes y juegos tradicionales, así como otros que sean creativos y pedagógicos" $(12,13)$.

Las preferencias que muestran los niños por los juegos dependen de diferentes factores, tales como el sexo, la edad cronológica y mental, la influencia familiar, las condiciones de vida y los valores de su entorno cultural. Las condiciones ideales se presentan en los niños de 7 a 12 años de edad por su actividad incansable y su espíritu emprendedor y creativo, que les permiten concentrarse y captar con prontitud todo lo relacionado con su entorno social (14). Por ello, la utilización de juegos en la etapa escolar es de gran valor para el fomento de conductas que propicien el cuidado de sí mismos y la protección de la familia y la comunidad.

En Venezuela el dengue constituye un problema de salud pública. En 1947 se organizaron campañas dirigidas a eliminar el mosquito vector mediante la aplicación de dicloro-difenil tricloroetano (DDT) y se implementó un programa de estructura vertical que se extendió hasta el año 1958, cuando se interrumpió, entre otras causas, por problemas administrativos. En 1989 el dengue reapareció con un incremento considerable en el número de casos, principalmente entre los menores de 15 años de edad y los habitantes de la región centrooccidental del país, en particular en los estados de Aragua y Lara. Más tarde se extendió al resto del país.

A partir de 1977 se inició un programa de control del vector y de vigilancia, que entre sus actividades fundamentales contemplaba la educación de toda la población para que tomara parte en la eliminación y el tratamiento de los criaderos del mosquito. La participación comunitaria es fundamental en todas las fases del programa, por lo que la preparación de la sociedad debe comenzar desde la educación básica con la incorporación de los escolares como promotores de salud en su comunidad. La participación de los docentes y los escolares del estado de Aragua en la lucha contra el vector antes del período de lluvias en 1995 permitió reducir mucho el número de casos de dengue en esa época (15).

Para favorecer la incorporación de los alumnos de la educación básica a la lucha contra el dengue, en 1999 Arias y colaboradores le propusieron al Ministerio de Sanidad y Asistencia Social el juego denominado Jugando en salud: dengue, elaborado con el apoyo de la Organización Panamericana de la Salud y del Proyecto para el Control de Enfermedades Endémicas - del Ministerio de Sanidad y Asistencia Social y del Banco Mundial-, con vistas a utilizarlo en las escuelas del estado de Aragua (16). Este juego está basado en un enfoque constructivista, reflejo de la tendencia actual de enseñar y aprender las materias científicas mediante aplicaciones prácticas relacionadas con temas circundantes locales y nacionales- de mayor relevancia (17).

Este juego tiene por finalidad instruir a los escolares acerca del dengue en Venezuela, despertar su motivación, curiosidad y participación, y evaluar los conocimientos aprendidos (16). Además, mediante estas actividades de educación en pro de la salud se puede recolectar información acerca de cómo iniciar desde la escuela movimientos dirigidos a dos dos a cambiar el comportamiento de los miembros de la comunidad, para que asuman sus responsabilidades en torno a la prevención y al control del dengue.

El objetivo del presente estudio fue evaluar el juego denominado Jugando en salud: dengue como medio de enseñanza-aprendizaje que favorece la incorporación de los escolares de la segunda etapa de educación básica a las tareas de control del mosquito Aedes aegypti y de prevención del dengue en el municipio de Girardot, del Estado Aragua, Venezuela.

\section{MATERIALES Y MÉTODOS}

Se llevó a cabo un estudio cuasi experimental, con dos grupos de estudio y un grupo de control, mediante la realización de pruebas de conocimientos y destrezas antes y después de la aplicación del juego Jugando en salud: dengue. Por su diseño, este estudio tuvo las características de un ensayo en la comunidad.

La población de estudio estuvo constituida por los alumnos de la segunda etapa (sexto grado) de las escuelas básicas estatales del municipio de Girardot, Estado Aragua, que tuvieran tres o más secciones de sexto grado. De las 39 escuelas estatales con las que cuenta este municipio, 29 tienen tres o más secciones de sexto grado con un total aproximado de 3330 alumnos distribuidos en 111 salones.

Para la selección de las 9 escuelas que participarían en el estudio se utilizó un muestreo al azar sistemático con un intervalo de selección $K=3$ $(K=29 / 9)$. Para ello se utilizó la lista de escuelas facilitada por las autoridades educacionales del Estado Aragua, las cuales fueron numeradas en orden correlativo ascendente.

En cada una de las 9 escuelas seleccionadas se formaron 3 salones, y los maestros seleccionaron al azar en cuál de sus tres salones se usaría el juego más el material teórico dado al maestro (salón no. 1), en cuál se usaría solo el material teórico dado al maestro (salón no. 2) y en cuál se mantendrían las actividades habituales (salón no. 3 o grupo de control). Finalmente, la muestra quedó formada por 621 estudiantes (18,6\% de la población total del sexto grado). De estos, 210 (33,8\%) formaron parte de los salones no. 1, 196 
(31,6\%) conformaron el grupo de salones no. 2 y 215 (34,6\%) fueron integrantes de los salones no. 3 .

En reunión con todos los alumnos de cada salón se explicó el objetivo de la investigación y se solicitó que participaran en una evaluación previa, con vistas a medir los conocimientos sobre el dengue antes de la intervención. A los maestros de los salones nos. 1 y 2 se les explicó que podían decidir las sesiones de clase y el tiempo que dedicarían a las actividades programadas, así como la forma de articular el juego con la dinámica de su clase.

Después de la evaluación inicial, cada maestro practicó el juego con sus alumnos tres veces a la semana durante 60 días en el salón no. 1. En el salón no. 2 los maestros desarrollaron las clases utilizando el contenido teórico del material de apoyo entregado. Al cabo del período de desarrollo de las actividades programadas, se aplicó la prueba de evaluación final en todos los salones.

Para el juego ese usan 20 tarjetas azules sobre comportamientos en torno al dengue, 40 tarjetas amarillas con preguntas fáciles, 40 tarjetas rojas con preguntas difíciles, un tablero de juego, un tablero para colocar las tarjetas sobre los comportamientos, 6 peones y una ruleta con puntero. Además de las instrucciones, el juego tiene una guía de orientación para el maestro con información sobre el dengue, ilustraciones sobre la estructura biológica del vector, indicaciones sobre las tareas y actividades que deben realizar los estudiantes y un glosario.

Las evaluaciones se realizaron mediante un cuestionario validado previamente. La validación se realizó en tres escuelas estatales del mismo municipio que fueron seleccionadas al azar entre las que no participarían en el estudio. Adicionalmente, el cuestionario se sometió a una prueba de expertos, lo que permitió ajustar su contenido y diseño. Al final quedó integrado por tres partes (anexo).

La primera parte del cuestionario estaba destinada a evaluar la aceptación del juego (dominio afectivo) por parte de los alumnos mediante cinco preguntas que valían de 1 a 5 puntos en una escala de gradación de Likert (puntuación máxima: 25; puntuación mínima: 5).

La segunda parte, dirigida a evaluar los conocimientos (dominio cognoscitivo), constaba de 10 preguntas cerradas de selección simple con un valor de un punto por cada respuesta correcta. Los resultados de esta parte del cuestionario permitieron evaluar la utilidad del juego como medio de enseñanza.

La tercera parte del cuestionario evaluó destrezas (dominio psicomotor) mediante seis preguntas, cada una con un valor de 1 a 5 puntos en una escala de gradación de Likert. Los resultados brindaron información adicional acerca de lo aprendido por los alumnos, la comprensión del material y su aplicación en actividades prácticas (18)

La información obtenida durante las evaluaciones inicial y final se procesó mediante una base de datos elaborada con el programa Statistix, versión 96. Para el análisis de los datos de distintos salones se calcularon las medidas de concentración y dispersión. Se utilizó el análisis de la varianza de clasificación simple para comparar los puntajes promedio de los niños en cada salón y establecer si existían diferencias significativas entre los grupos en cuanto a sus conocimientos y destrezas o habilidades. Una vez encontradas diferencias estadísticamente significativas entre los grupos, se aplicó la prueba de Scheffé para determinar diferencias dentro de los grupos (19). El nivel de significación escogido fue de 0,05 .

\section{RESULTADOS}

Las 9 escuelas seleccionadas estaban distribuidas en cuatro de las ocho parroquias que tiene el municipio de Girardot.

La edad de los escolares que participaron en la investigación fue de 8 a 16 años (media: 11,8 años), según lo contemplado por el Ministerio de Educación, Cultura y Deportes para la segunda etapa de la educación básica. La relación entre sexos fue aproximadamente de 1:1 (309 niñas y 312 niños), lo que coincide con los resultados de investigaciones similares (6-8). En general, los estudiantes consideraron aceptable el juego y $86,7 \%$ de ellos valoraron esta aceptación como alta o muy alta (cuadro 1).

La media general de la puntuación obtenida por cada grupo de salones antes de la aplicación del programa propuesto fue similar (entre 6,1 y 7,1 puntos), lo que demuestra la pobreza de los conocimientos básicos sobre el dengue que adquieren los estudiantes mediante los programas escolares.

$\mathrm{Al}$ analizar por escuela y por grupos de salones los conocimientos sobre el dengue que tenían los 621 escolares participantes antes de la aplicación del instrumento (prueba inicial) mediante el análisis de la varianza se observaron diferencias significativas $(P<0,05)$ entre los grupos (cuadro 2). La comparación por el método de Scheffé de las puntuaciones medias de los distintos salones reveló diferencias significativas entre el salón no. 3 y los salones nos. 1 y 2.

CUADRO 1. Grado de aceptación del juego. Escuelas básicas estatales del municipio de Girardot, estado de Aragua, 2000

\begin{tabular}{lcc}
\hline Grado de aceptación & No. & $\%$ \\
\hline No aceptado & 4 & 1,9 \\
Aceptación baja & 5 & 2,4 \\
Aceptación media & 19 & 9,0 \\
Aceptación alta & 86 & 41,0 \\
Aceptación muy alta & 96 & 45,7 \\
$\quad$ Total & 210 & 100,0 \\
\hline Media = 22,3 puntos; mediana = 23,0 puntos; desviación estándar = 3,2.
\end{tabular}


Debido a la ausencia de varios estudiantes de la muestra, los conocimientos obtenidos mediante el juego fueron evaluados solo en 597 alumnos. Según los resultados de la prueba final, el grupo de salones no. 1 elevó su puntuación media de 6,1 a 9,2 ( $P<0,05)$; el grupo de salones no. 2 la aumentó de 6,1 a $8,2(P<0,05)$ y el grupo de salones no. 3 solo aumento su puntuación media de 7,1 a 7,3 $(P>$ $0,05)$, lo que evidenció un mayor aprendizaje en los grupos que utilizaron el juego más el material teórico (grupos de salones No. 1) y el material teórico solamente (grupo de salones no. 2) que en el grupo de salones no. 3, que fue el grupo de control (cuadros 2 y 3). Según el método de Scheffé, los tres grupos difirieron entre sí, con un valor crítico de $F=3,011(P<0,05)$.

Las destrezas de los escolares para controlar el vector $A$. aegypti (cuadro 4) antes del ejercicio propuesto fueron similares en todas las escuelas, con una puntuación media de 18,4 (puntuación mínima: 16,9; puntuación máxima: 19,7). No obstante, al evaluar las destrezas alcanzadas después del ejercicio, la puntuación promedio se elevó a 23,0 (puntuación mínima: 20,7; puntuación máxima: 25,8), lo cual refleja un aumento de las habilidades para el control del vector $A$. aegypti ( $P$ $<0,05)$.

El análisis de la varianza reveló la existencia de diferencias significativas entre los grupos de salones en cuanto a las destrezas de los escolares antes de la intervención $(P<0,05)$. Mediante el método de Scheffé se encontró que el grupo de salones no. 1 tenía mayores habilidades que los grupos de salones nos. 2 y 3 , con un valor crítico de $F=3,011(P<0,05)$.

No obstante, en la evaluación posterior a la aplicación del juego (cuadros 5 y 6) se observó un aumento considerable de la puntuación promedio obtenida por los estudiantes en todos los grupos de salones en su conjunto, y puede afirmarse que el nivel de habilidades aprendidas y desplegadas por los estudiantes mejoró en grado significativo $(P<0,05)$ en los salones nos. 1 y 2 en comparación con el no. 3 .

Al verificar las diferencias entre los grupos mediante la comparación de medias se observó que los grupos de

CUADRO 2. Resultados (medias aritméticas) de las pruebas de conocimiento por grupos de salones. Escuelas básicas estatales del municipio de Girardot, Estado Aragua, 2000

\begin{tabular}{|c|c|c|c|c|c|c|}
\hline \multirow[b]{2}{*}{ Escuelas } & \multicolumn{2}{|c|}{ Salón no. 1} & \multicolumn{2}{|c|}{ Salón no. 2} & \multicolumn{2}{|c|}{ Salón no. 3} \\
\hline & $\begin{array}{c}\text { Prueba } \\
\text { inicial }\end{array}$ & $\begin{array}{c}\text { Prueba } \\
\text { final }\end{array}$ & $\begin{array}{c}\text { Prueba } \\
\text { inicial }\end{array}$ & $\begin{array}{c}\text { Prueba } \\
\text { final }\end{array}$ & $\begin{array}{c}\text { Prueba } \\
\text { inicial }\end{array}$ & $\begin{array}{c}\text { Prueba } \\
\text { final }\end{array}$ \\
\hline No. 1 & 6,1 & 10,0 & 6,0 & 8,2 & 5,5 & 6,1 \\
\hline No. 2 & 5,7 & 8,6 & 5,3 & 7,3 & 5,8 & 5,6 \\
\hline No. $3^{a}$ & 5,8 & 9,8 & 7,3 & $\ldots$ & 8,0 & 7,6 \\
\hline No. 4 & 6,6 & 9,1 & 6,6 & 8,4 & 6,2 & 7,1 \\
\hline No. 5 & 5,1 & 8,4 & 5,5 & 9,5 & 6,7 & 8,6 \\
\hline No. 6 & 6,3 & 7,4 & 6,7 & 8,2 & 9,4 & 10,0 \\
\hline No. 7 & 5,9 & 8,9 & 5,9 & 8,1 & 7,1 & 6,2 \\
\hline No. 8 & 5,4 & 9,8 & 6,0 & 8,8 & 7,8 & 8,5 \\
\hline No. 9 & 8,5 & 10,0 & 5,1 & 8,3 & 7,1 & 5,5 \\
\hline Total $^{*}$ & 6,1 & 9,2 & 6,1 & 8,2 & 7,1 & 7,3 \\
\hline
\end{tabular}

a El salón no. 2 de esta escuela declinó continuar en la investigación y no realizó la prueba final.

CUADRO 3. Medidas descriptivas de las pruebas de conocimiento realizadas, por grupos de salones. Escuelas básicas estatales del municipio de Girardot, Estado Aragua, 2000

\begin{tabular}{lccccccc}
\hline & \multicolumn{3}{c}{ Prueba inicial } & & \multicolumn{3}{c}{ Prueba final } \\
\cline { 2 - 4 } Grupos & $n$ & $X$ & & & & & \\
\hline Salones no. 1a & 197 & 6,13 & 1,61 & & 210 & 9,28 & 1,54 \\
Salones no. 2 & 196 & 6,09 & 1,63 & & 174 & 8,24 & 1,27 \\
Salones no. 3 & 215 & 7,13 & 2,04 & & 213 & 7,33 & 2,24 \\
\hline
\end{tabular}

Nota: X: media aritmética; DE: desviación estándar.

a 13 estudiantes no se presentaron a la escuela el día de la prueba inicial, pero se incorporaron posteriormente a las actividades educativas y a la prueba final.

CUADRO 4. Medias de las destrezas previa y posterior observadas en los escolares. Escuelas básicas estatales del municipio de Girardot, Estado Aragua, 2000

\begin{tabular}{lcc}
\hline Escuelas & Destreza previa & Destreza posterior \\
\hline No. 1 & 18,9 & 22,6 \\
No. 2 & 19,2 & 24,0 \\
No. 3 & 18,8 & 20,7 \\
No. 4 & 18,6 & 23,6 \\
No. 5 & 18,2 & 25,8 \\
No. 6 & 18,7 & 22,0 \\
No. 7 & 17,1 & 22,1 \\
No. 8 & 16,9 & 23,2 \\
No. 9 & 19,7 & 23,7 \\
Total & 18,4 & 23,0 \\
\hline
\end{tabular}

salones nos. 1 y 2 no difirieron entre sí (valor critico de $F=3,011 ; P<0,05$ ), lo que puede reflejar la importancia del material teórico proporcionado a los maestros en ambos grupos. Sin embargo, se encontró que los estudiantes de los grupos de salones nos. 1 y 2 incorporaron más destrezas y habilidades que los del grupo de control.

\section{DISCUSIÓN}

El diseño de las pruebas de evaluación, basadas en la selección de una respuesta entre varias opciones, 
CUADRO 5. Resultados (medias aritméticas) de las pruebas de destrezas por grupos de salones. Escuelas básicas estatales del municipio de Girardot, Estado Aragua, 2000

\begin{tabular}{|c|c|c|c|c|c|c|}
\hline \multirow[b]{2}{*}{ Escuelas } & \multicolumn{2}{|c|}{ Salón no. 1} & \multicolumn{2}{|c|}{ Salón no. 2} & \multicolumn{2}{|c|}{ Salón no. 3} \\
\hline & $\begin{array}{c}\text { Prueba } \\
\text { inicial }\end{array}$ & $\begin{array}{c}\text { Prueba } \\
\text { final }\end{array}$ & $\begin{array}{c}\text { Prueba } \\
\text { inicial }\end{array}$ & $\begin{array}{c}\text { Prueba } \\
\text { final }\end{array}$ & $\begin{array}{c}\text { Prueba } \\
\text { inicial }\end{array}$ & $\begin{array}{c}\text { Prueba } \\
\text { final }\end{array}$ \\
\hline No. 1 & 20,5 & 21,9 & 18,1 & 24,4 & 18,0 & 21,8 \\
\hline No. 2 & 20,3 & 24,7 & 17,5 & 24,1 & 19,5 & 23,2 \\
\hline No. $3^{a}$ & 20,2 & 22,0 & 19,1 & $\ldots$ & 17,3 & 19,7 \\
\hline No. 4 & 21,8 & 25,6 & 16,7 & 23,4 & 17,7 & 21,4 \\
\hline No. 5 & 20,3 & 26,4 & 16,4 & 24,1 & 17,9 & 26,4 \\
\hline No. 6 & 19,3 & 23,3 & 18,8 & 22,8 & 18,1 & 20,7 \\
\hline No. 7 & 17,2 & 22,1 & 17,6 & 22,5 & 16,5 & 21,6 \\
\hline No. 8 & 16,0 & 22,6 & 18,9 & 23,3 & 15,9 & 23,9 \\
\hline No. 9 & 20,7 & 27,2 & 19,2 & 22,2 & 19,2 & 21,6 \\
\hline Total & 19,5 & 23,6 & 18,1 & 23,3 & 17,7 & 22,0 \\
\hline
\end{tabular}

a El salón no. 2 de esta escuela declinó continuar en la investigación y no realizó la prueba final.

CUADRO 6. Medidas descriptivas de las pruebas de destreza realizadas, por grupos de salones. Escuelas básicas estatales del municipio de Girardot, Estado Aragua, 2000

\begin{tabular}{|c|c|c|c|c|c|c|}
\hline \multirow[b]{2}{*}{ Grupos } & \multicolumn{3}{|c|}{ Prueba inicial } & \multicolumn{3}{|c|}{ Prueba final } \\
\hline & $n$ & $X$ & $\mathrm{DE}$ & $n$ & $X$ & DE \\
\hline Juego (Salones no. 1)a & 197 & 19,52 & 4,56 & 210 & 23,62 & 4,47 \\
\hline Teoría (Salones no. 2) & 196 & 18,09 & 4,44 & 174 & 23,32 & 4,54 \\
\hline Control (Salones no. 3) & 215 & 17,73 & 3,52 & 213 & 22,04 & 4,49 \\
\hline
\end{tabular}

Nota: X: media, DE: desviación estándar.

13 estudiantes no se presentaron a la escuela el día de la prueba inicial, pero se incorporaron posteriormente a las actividades educativas y a la prueba final.

permitió lograr una mayor uniformidad y confiabilidad en las mediciones realizadas, además de facilitar la codificación y el análisis de los resultados. La primera parte de la evaluación estuvo dirigida a conocer la opinión de los alumnos sobre el diseño, las instrucciones y las preguntas del juego, así como su facilidad y el tiempo dedicado a jugarlo. En general, los estudiantes consideraron aceptable el juego y $86,7 \%$ de ellos valoraron esta aceptación como alta o muy alta.

La segunda y tercera partes del cuestionario estaban dirigidas a explorar los conocimientos y destrezas de los estudiantes, respectivamente. La combinación de las preguntas de selección con preguntas abiertas en la primera y tercera partes del cuestionario permitió obtener mejores resultados (20).

En el programa para la educación básica se destaca que el juego, además de impulsar la formación del pensamiento simbólico en el educando, favorece la comunicación y el desarrollo del lenguaje, incentiva la satisfacción por la búsqueda y la experimentación, y permite el dominio natural del espacio y del tiempo. Por ello, el juego puede ayudar a promover la salud y prevenir enfermedades, como el dengue, mediante el fomento de conductas responsables en la población (14).

A pesar de que desde 1989 cuando se presentó la primera epidemia de dengue en Venezuela, en particular, en el estado de Aragua- el plan de estudios de la educación básica contempla el tema de la prevención y el control del dengue, el conocimiento inicial que mostraron los diferentes grupos estudiados fue reducido $y$ variable, posiblemente debido a la influencia de otras fuentes de información fuera del ambiente escolar (7).
Si bien el material teórico proporcionado al maestro permitió aumentar la puntuación media alcanzada inicialmente por el grupo de salones no. 2, el avance del grupo de salones no. 1 fue muy superior, lo que indica que el juego constituye un medio educativo más eficaz.

El grupo de salones no. 3, utilizado como grupo de control, no presentó cambios significativos en la media de su puntuación antes y después de la aplicación del programa propuesto, a pesar de que en el momento de la investigación los maestros presentaban el tema del dengue como parte del programa de estudio habitual.

Estos resultados confirman que el juego, como medio educativo, favorece la comprensión y mejora la calidad y cantidad de los conocimientos aprendidos $(3,9,10)$, pues la interacción, el diálogo oral, la observación y la experimentación contribuyen al desarrollo de los educandos y mejoran su capacidad de argumentación y su comportamiento. Además, los resultados de este trabajo reafirman que el juego combinado con un material teórico proporcionado a los maestros a manera de apoyo rinde mejores resultados que solo el contenido teórico, y ambos, por separado, superan la práctica docente actual.

Las diferencias en los conocimientos adquiridos por los estudiantes, demostrada mediante la comparación de las puntuaciones medias antes y después de las intervenciones descritas, indican que el juego Jugando en salud: dengue es un medio educativo de gran valor para la prevención del dengue. Dotar de buenos conocimientos teóricos al maestro permitió enriquecer el proceso de enseñanza-aprendizaje, que va más allá de la clase tradicional. Esto debe estimular a los profesionales de la salud a diseñar y elaborar materiales teóricos actualizados dirigidos a los maestros, con el fin de promover la participación de los estudiantes en la prevención $y$ el control de enfermedades como el dengue.

Por su parte, los maestros deben estar preparados para utilizar juegos en las aulas y lograr la participación de los alumnos. La selección de estrategias metodológicas adecuadas para el aprendizaje depende de la capacidad del educador para aplicarlas, ya que los medios de 
enseñanza por sí solos no son suficientes para lograr los objetivos propuestos (3). En este sentido, ha quedado demostrado que cuando a un maestro se le suministra material didáctico bien elaborado y estructurado en forma de juego para que lo aplique como parte de un proceso docente dinámico, se puede motivar a los estudiantes en la búsqueda y adquisición de conocimientos. De esta forma se logra formar estudiantes comprometidos con su propia salud y con su entorno.

Las diferencias encontradas entre los grupos de salones demuestran que el juego Jugando en salud: dengue, como medio educativo en la enseñanza básica, facilita la incorporación de habilidades y destrezas para combatir el dengue y su vector, a la vez que facilita la interacción y el diálogo en los educandos. De esta forma, ayuda a desarrollar en ellos la capacidad de explicar su conducta frente a situaciones que exigen su atención, así como actitudes dirigidas a proteger su salud.

\section{CONCLUSIONES}

Los resultados de la evaluación realizada demuestran que el juego tuvo muy buena aceptación entre los estudiantes de la segunda etapa de la enseñanza básica. Les permitió adquirir más conocimientos sobre el dengue y desarrollar habilidades y destrezas que favorecieron su incorporación a actividades de prevención de la enfermedad en sus respectivas comunidades. Se recomienda extender la utilización de este juego a todas las escuelas básicas como una vía para fortalecer el proceso educativo e incorporar a los niños a las actividades de control del dengue.

ANEXO. Contenido del instrumento evaluador del programa educativo propuesto. Las partes primera y tercera se evaluaron mediante una escala de gradación de Likert

Primera parte: nivel de aceptación

A. El diseño del juego

B. Las instrucciones del juego

C. Las preguntas del juego

D. La facilidad del juego

E. El tiempo de juego

¿Cuántas veces jugaste el juego?

Escala de respuestas: No sé, me disgusta mucho, no me gusta, me gusta y me gusta mucho.

\section{Segunda parte: nivel de conocimiento}

1. El dengue es una enfermedad transmisible producida por:

- un ratón

- un virus

- un ave

2. Los síntomas de una persona que tiene dengue son:

- fiebre, escalofrío y sed

- fiebre, sed y erupción

- fiebre, escalofrío y erupción

3. Los tipos de dengue que conoces son:

- dengue y dengue hemorrágico

- dengue papera y dengue

- dengue hemorrágico y dengue polio

4. El transmisor del dengue es:

- el mosquito Aedes aegypti

- el chipo Rhodnius prolixus

- el mosquito Culex

5. El ciclo del mosquito desde huevo hasta adulto dura entre:

- 1 año y unas semanas

- 3 meses y una semana

- 1 y 3 semanas
6. Los huevos de mosquito liberan:

- mariposas

- larvas

- gusanos

7. El mosquito Aedes aegypti se cría en:

- jaulas

- agua limpia

- aire

8. La responsabilidad de controlar el dengue es de:

- el maestro

- el gobierno

- todos nosotros

9. Los métodos de protección individual contra el dengue incluyen

- uso de mosquiteros y repelentes

- higiene personal y agua estancada

- repelente y basura

10. Para prevenir el dengue debemos:

- permitir que nos pique un zancudo

- tapar los envases que almacenan el agua que usamos

- criar los zancudos

\section{Tercera parte: nivel de destreza}

1. ¿Limpias el patio de tu casa?

2. ¿Cuándo realiza tu comunidad labores de limpieza en las que tú participas?

3. ¿Tu familia y tú mantienen limpios y libres de basura los alrededores de tu casa?

4. ¿Tapas los pipotes que almacenan agua?

5. ¿Utilizas repelentes de insectos?

6. ¿Tú botas el agua acumulada en los envases y objetos desechados que no usas?

¿Qué dirías del dengue?

Escala de respuestas: Nunca, un día a la semana, tres días a la semana, cinco días a la semana y todos los días de la semana. 


\section{REFERENCIAS}

1. Luzuriaga L. Pedagogía. Buenos Aires: Editorial Lozada; 1975.

2. Morles V. Pruebas de rendimiento escolar. Guía para su preparación, ejecución y análisis. Caracas: Fondo Editorial de Humanidades y Educación, UCV; 1972.

3. Montoya S. Educación en salud. En: Fundamentos de Salud Pública. Tomo I. Medellín, Colombia: Corporación para Investigaciones Biológicas; 1997.

4. Rivas Balboa C. Un nuevo paradigma en educación y formación de recursos humanos. Cuadernos Lagoven. Caracas: Editorial Arte; 1996.

5. Venezuela, Ministerio de Educación, Cultura y Deportes. Programa educativo vigente. Caracas: Fondo Editorial del Ministerio de Educación; 1998.

6. Fabbricatore $\mathrm{O}$, Méndez E, Rubio $\mathrm{M}$. Currículo en educación básica. Análisis de caso: Venezuela. Disponible en http://www. monografias.com/trabajos11/cued/cued.sht ml. Acceso el 24 de noviembre de 2003.

7. Melet N. Una estrategia metodológica para la enseñanza de la educación para la salud. Valencia, Venezuela: Ediciones Universidad de Carabobo; 1988.
8. Santos M, Magalhaes T, Bittencourt P. Playing and learning-bate-boca. An educational game concerning schistosomiasis, AIDS, dengue and leishmaniasis. Cien Cult (São Paulo) 1993:45(6):381-385. (Lilacs/CD-R33/203434).

9. Soto R, Fernández E, Ávila G. Evaluación de un programa educativo sobre dengue y Aedes aegypti focalizado en niños de escuela primaria. Rev Med Honduras 1995;63(1):12-18. (Lilacs/CD-R 33157129).

10. Díaz Vega J. El juego y el juguete en el desarrollo del niño. México, D.F.: Editorial Trillas; 1997.

11. Fingerman G. El juego y sus predicciones sociales. Buenos Aires: Editorial El Ateneo; 1970

12. Venezuela, Asamblea Nacional Constituyente. Constitución de la República Bolivariana de Venezuela. Gaceta Oficial de la Republica Bolivariana de Venezuela 2000, marzo 24. (Número 5453, extraordinario).

13. Ley Orgánica para la Protección del Niño y del Adolescente. Gaceta Oficial de Venezuela 1998, octubre 11. (No. 5266, extraordinario).

14. Oppenheim J. Los juegos infantiles. Barcelona: Ediciones Martínez Roca; 1990.

15. Venezuela, Ministerio de Sanidad y Asistencia Social, Organización Panamericana de la Salud. El dengue y el sarampión en
Venezuela. Temas de epidemiología Caracas: OPS/OMS, MSAS; 1997.

16. Arias J, Guevara M, Vivas E. Jugando en salud: dengue. Proyecto de Control de Enfermedades Endémicas OPS/PCEE (Ministerio de Sanidad y Asistencia SocialBanco Mundial). Maracay, Venezuela: Escuela de Malariología y Saneamiento Ambiental Dr. Arnoldo Gabaldón; 1999.

17. Rivas C. Un nuevo paradigma en educación y formación de recursos humanos. Cuadernos Lagoven. Caracas: Editorial Arte; 1996.

18. Beyer B. Enseñar a pensar. Libro guía para docentes. 1. ${ }^{a}$ ed. Buenos Aires: Editorial Troquel; 1998.

19. Taucher E. Bioestadística. Santiago, Chile: Editorial Universitaria; 1997.

20.Kerlinger F. Investigación del comportamiento. Técnicas y metodología. 2. ed. México, D.F.: Nueva Editorial Interamericana; 1986.

Manuscrito recibido el 24 de febrero de 2003. Aceptado para publicación, tras revisión, el 17 de septiembre de 2003.
ABSTRACT

\section{A game as an educational strategy for the control of Aedes aegypti in Venezuelan schoolchildren}

Objective. To assess the value of a game known as Jugando en salud: dengue [Playing for health: dengue] as a teaching/learning tool leading to the incorporation of schoolchildren enrolled in secondary school in the control of Aedes aegypti and dengue prevention activities in the municipality of Girardot, Aragua state, Venezuela.

Methods. We performed a descriptive, quasi-experimental study based on field research carried out in 9 schools that were chosen by systematic random sampling among the 29 public schools in the municipality of Girardot, Aragua state, Venezuela, having three or more sixth-grade classrooms. In each school three workrooms were set up: in group no. 1 (210 students) the game was practiced three times a week for a total of 60 days, and the teacher was furnished with theoretical materials about dengue; in group no. 2 (196 students) only the theoretical materials that were given to the teacher were used, and in group no. 3 (215 students), which was the control group, the regular learning program established by the Ministry of Education was followed. Participating students were evaluated before and after each of the proposed programs by means of a questionnaire that was evaluated using a Likert scale. We calculated concentration and dispersion measures for the data obtained during the evaluations. Simple analysis of variance was used to compare the mean results obtained in the different classrooms and to detect significant differences among the various groups in terms of knowledge and skills before and after the proposed program. Scheffé's test was used to detect differences within groups.

Results. Six hundred twenty-one schoolchildren between the ages of 8 and 16 years (mean age: 11.8 years) participated in the study. Of the 210 students that took part in the game, $41 \%$ rated its acceptability as "high" and $45 \%$ as "very high." The knowledge about dengue and the skills that were measured prior to following the prescribed program were lower (6.5 and 18.4 points, respectively) than those displayed in the final test (8.25 and 22.9 points, respectively; $P<0,05)$. There appeared to be better learning in the groups that used the game plus the theoretical materials (group no. 1), or that used just the theoretical materials (group no. 2), than in the control group (group no. 3). Students in workrooms 1 and 2 also appeared to acquire more skills than those in the control group $(P<0,05)$.

Conclusions. The game was highly accepted among students enrolled in secondary school. It helped them acquire greater knowledge about dengue and to develop skills and abilities leading to their incorporation in dengue prevention activities in their respective communities. We recommend that the game be extended to all schools as a tool for strengthening the educational process and incorporating children in dengue control activities. 